\title{
Electromagnetic analysis of UHF Near-field RFID Tag Antenna
}

\author{
M. Dhaouadi ${ }^{1}$, M. Mabrouk ${ }^{1}$, IEEE Member, A. Ghazel ${ }^{1}$, IEEE Senior Member and \\ S. Tedjini', IEEE Senior Member
}

\begin{abstract}
${ }^{1}$ CIRTACOM, SUPCOM-ISETCOM de Tunis, University of Carthage, Cité Technologique des Communications, 2088, Tunisia
\end{abstract}

${ }^{2}$ ORSYS, LCIS, ESISAR-INPG, 50 rue de Laffemas, BP 54, 26902, Valence Cedex 9, France

mondher.dhaouadi@supcom.rnu.tn

mohamed.mabrouk@isetcom.rnu.tn

\begin{abstract}
In this paper, the performance of Near-Field UHF RFID systems is investigated by means of electromagnetic analyses. A novel antenna is presented for ultra high frequency (UHF) near-field radio frequency identification (RFID) applications. The reactance component of tag antenna considered chip impedance (-193j) is conjugated and matched for maximum power transmission. The antenna is fabricated by using Polyester (PET) dielectric substrate with permittivity 3.2. The size of antenna is $38 \times 18 \times 0.5 \mathrm{~mm}$. The HFSS simulator is used for optimizing the proposed antenna. The antenna Return Loss is of $14.0 \mathrm{~dB}$ for the worst case in the middle of UHF range, better than $31.4 \mathrm{~dB}$ at $900 \mathrm{MHz}$
\end{abstract}

\section{Introduction}

The RFID (Radio Frequency Identification) is a non-contact method for data transfer to object identification [1]. Two major characteristics that distinguish different types of RFID systems are the power source of the tag and the frequency of operation [2]. RFID can be categorized into two types: an active RFID capable of transmitting an electromagnetic wave containing information of the RFID, and a passive RFID which drives utilizing power of an electromagnetic wave externally received. A strictly passive RFID system uses the energy from the field radiated by the reader to completely power the tags. The tag then uses this energy to identify itself and communicate with the reader. The basic near field UHF RFID concept is to make UHF RFID system work at short distances and on different objects as reliably as LF/HF RFID [3]. Near-field UHF technology is capable of transmitting in the nearfield, similar to HF, but is faster and works well around metals and liquids [4]. The aim of this work is to investigate the electromagnetic characteristics of UHF near field RFID Tag Antenna.

\section{Near-field UHF RFID Tag}

Near field RFID are based on inductive coupling between the reader and the Tag. UHF near-field RFID is increasingly popular for item-level tagging because the tag can be detected more consistently on various objects such as bottles of water, CD/DVD, and small items [5]. Since the major part of reactive energy is stored in the magnetic field, the system of inductive coupling is preferred for the communication between the antenna of reader and the antenna of Tag. Most of the near field UHF RFID Tags antennas are rectangular or circular loops, as these electrically small loop antennas are able to produce a strong and uniform magnetic field in the region around the antenna. Finally, to have a good magnetic coupling in near field UHF RFID Tag antenna, it is necessary to use Tmatch configuration with a circular or rectangular for tags antennas [6].

\section{ANTENNA DESIGN}

Fig. 1 shows the proposed tag antenna structure. The antenna is fabricated with a flexible polyester (PET) substrate (thickness $H=50 \mathrm{~m}$, relative dielectric constant $\varepsilon_{r}=3.2$, and loss tangent $\tan \delta=0.003$ ) with an overall size of $38 \times 18 \mathrm{~mm}^{2}$. The detailed dimensions of the antenna prototype are: $L_{1}=13.9 \mathrm{~mm}, L_{2}=6.95 \mathrm{~mm}$, 
$W_{1}=1 \mathrm{~mm}, W_{2}=1.08 \mathrm{~mm}$ and $W_{3}=2 \mathrm{~mm}$. The operation frequency of the tag is in the UHF band. For the purpose of design calculations, the frequency of $900 \mathrm{MHz}$ has been chosen.

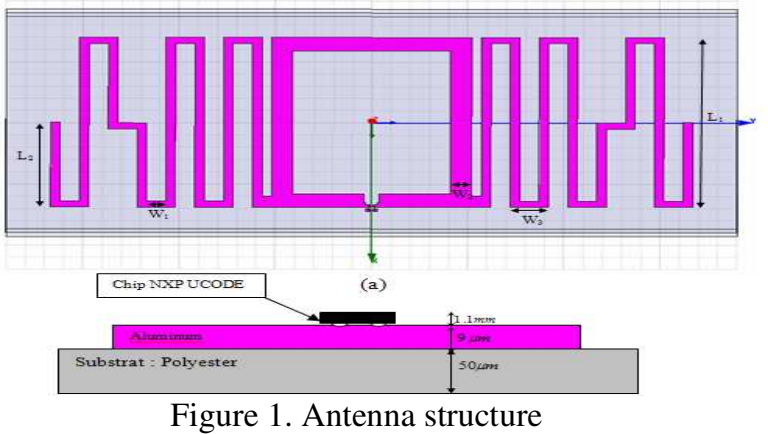

(a) Top view (b) Side view

Integrated chip ASIC Philips (NXP UCODE) enclosed in TSSOP8 body was used for the tag [7]. For maximum power transfer between the tag antenna and the RFID IC, the tag antenna impedance must be the complex conjugate of the RFID IC impedance. The RFID IC impedance is highly capacitive in nature the conjugate match in predominantly inductive. In this paper the antenna is designed for a tag chip with $Z_{c}=\left(\begin{array}{ll}22 & j 193\end{array}\right) \Omega$ at a resonant frequency of $900 \mathrm{MHz}$. The load antenna impedance should be $Z_{a}=(22+j 193) \Omega$ for conjugate matching and to transmit the maximum power between the antenna and the microchip.

\section{Results}

\subsection{Antenna Performance}

The antenna was designed using electromagnetic simulation tool Ansoft HFSS which allowed us to calculate antenna gain, impedance, and proper matching to the RFID chip. The return loss $-31.4 \mathrm{~dB}$ is obtained at the frequency $900 \mathrm{MHz}$ is shown in Fig.2.

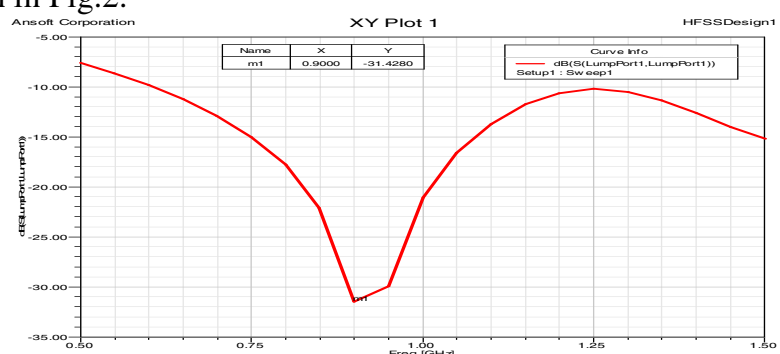

Figure 2. Simulated Antenna Return Loss

Fig.3 shows the predicted VSWR performance of the antenna vs frequency (at $900 \mathrm{MHz}$ ).

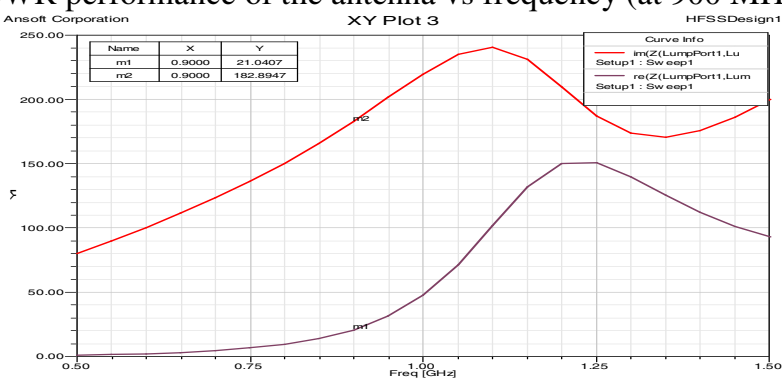

Figure 3. Antenna impedance against frequency: Resistance component $R_{a}$, Reactance component $X_{a}$

The Voltage Standing Wave Ratio (VSWR) of antenna is another characteristic parameter used to measure the impedance matching of an antenna to its connected load. It is defined as the ratio of the reflected voltage over the incident voltage. Fig. 4 shows that for a VSWR of $0.46 \mathrm{~dB}$. 


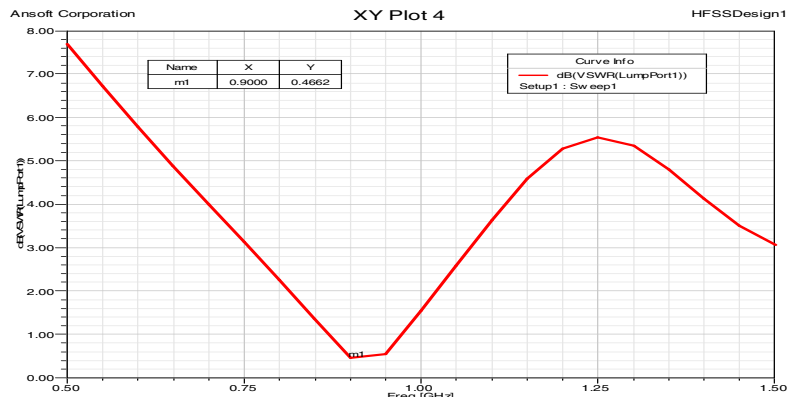

Figure 4. Antenna VSWR vs. frequency

\subsection{Field Analysis of Tag antenna}

In order to analyze the performance in the near zone, the electric field distribution is simulated in horizontal plane. Electric fields are created by differences in voltage: the higher the voltage, the stronger will be the resultant field. Fig. 5 illustrates electric field distribution in the near-field RFID Tag antenna shown in Fig. 1. The electric fields are strongest close to their origin (chip) and rapidly decrease at greater distances from the chip NXP (source).

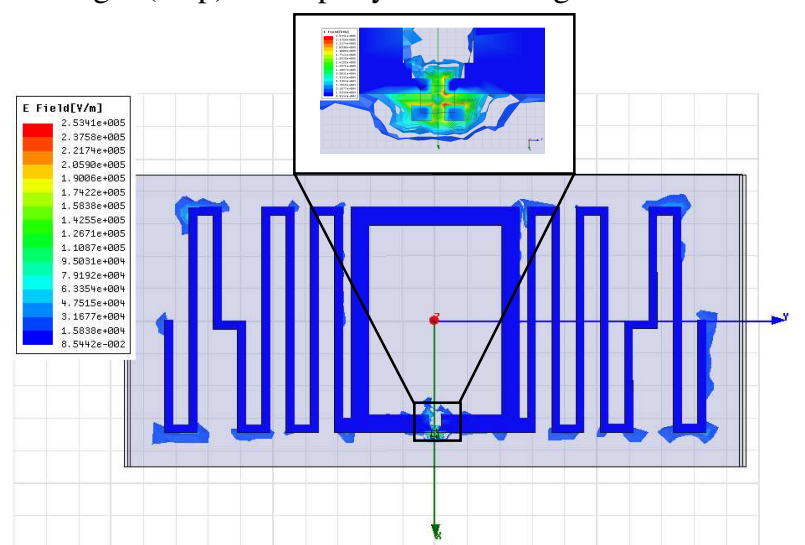

Figure 5. Electric field plot of the UHF near field RFID Tag Antenna

Fig. 6 shows the simulated magnetic field distribution in the near-field RFID Tag antenna.

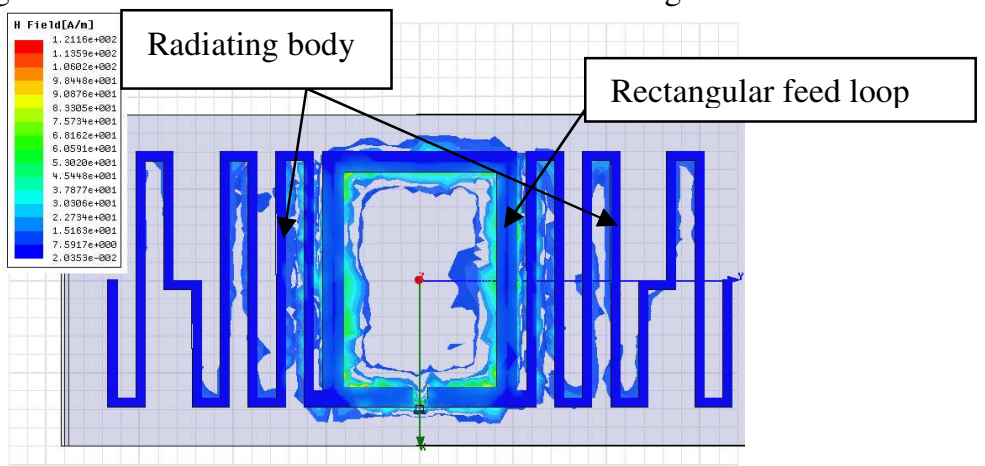

Figure 6. Magnetic field plot of the UHF near field RFID Tag Antenna

The current on a side of the rectangular feed loop is not same magnitude as that on other side of the radiating body but it is reduced progressively. The current fluctuation generates a variation of magnetic field. Then the strength of the magnetic field decreases with the diminution of the current. The reduction in the magnetic field produces a distribution of this field which is not uniform for near-field RFID applications. 


\subsection{Placing antenna Tag RFID in metal environment}

It is expected that the thin metal foil will reduce the performance of the RFID tag [8]. Metal introduces difficulties for antennas in systems using radio communication in the far field as well as for antennas in inductively coupled systems working in the near field. Fig.7 illustrates the influence of a metal plate on the radiation pattern of antenna Tag RFID.
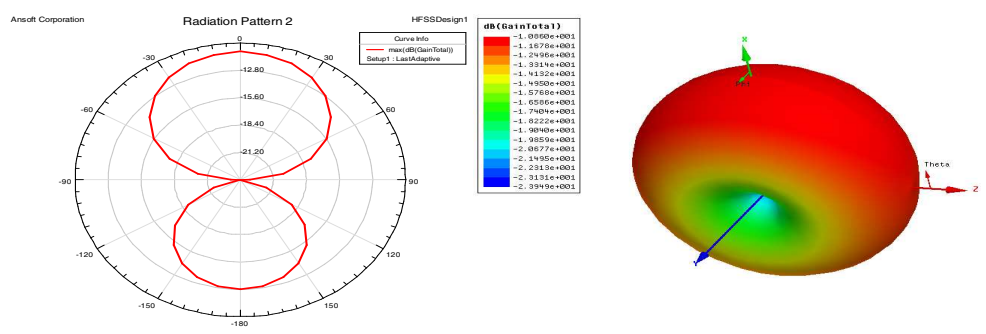

Figure7. Simulated directivity pattern of the tag antenna structure located in free space
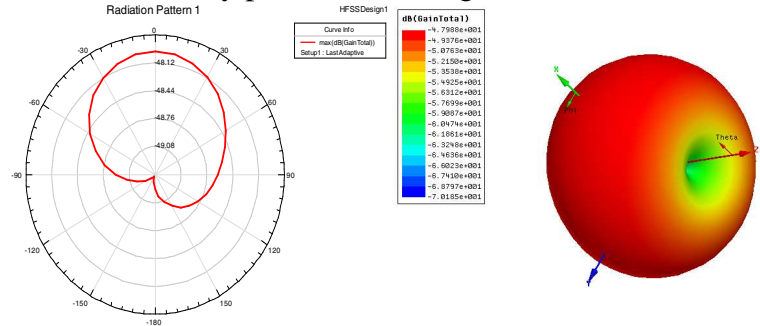

Figure 8 . Simulated directivity pattern of the tag antenna structure located in metallic environments

A magnetic field cannot penetrate metal or other magnetically conductive materials. The course of the lines of electric flux is changed and has therefore a considerable influence on the radiation pattern.

\section{Conclusion}

In this paper, the performance of Near-Field UHF RFID systems is investigated by means of electromagnetic analyses. We presented a novel planar RFID Tag antenna for near-zone UHF communication. Besides the space limitation, we verified that the thin metal foil will reduce the performance of the RFID tag.

\section{References}

1. R.Schneiderman, "RFID Tags Locate Growing Wireless Markets", Microwaves \& RF Magazine, Feb.1994.

2. K. Finkenzeller, "RFID Handbook: Fundamentals and Applications in Contactless Smart Cards and Identification", John Wiley and Sons, England, 2003.

3. Nikitin, P.V. Rao, K.V.S. Lazar, S. “An Overview of Near Field UHF RFID”, IEEE International Conference on RFID, 2007.

4. Claire Swedberg, "A Shift to UHF Near-Field Predicted for Pharma", RFID Journal, 2005, www.rfidjournal.com/article/.../rfidjournal-article2694.PDF.

5. A. Shameli, A. Safarian, A. Rofougaran, J. Castaneda, and F. De Flaviis, "A UHF near field RFID system with fully integrated transponder," IEEE Trans. Microw. Theory Tech., vol. 56, pp.1267-1277, May 2007.

6. M.Dhaouadi, M.Mabrouk, A.Ghazel, "Magnetic antenna for near-field UHF RFID tag",18 th International conference on Microwave, Radar and Wireless Communications MIKON-2010, June 2010.

7. UCODE G2XM and UCODE G2XL (TSOOP8 Package Specification) from December 2007, http://www.nxp.com/ .

8. Byunggil Yu, Sung-Joo Kim, Byungwoon Jung, Harackiewicz F.J, Myun-Joo Park, Byungje Lee, "Balanced RFID Tag Antenna Mountable on Metallic Plates", Antennas and Propagation Society International Symposium, IEEE, July 2006. 